\title{
Automated and Scalable T-wise Test Case Generation Strategies for Software Product Lines
}

\author{
Gilles Perrouin ${ }^{1}$, Sagar Sen ${ }^{2}$, Jacques Klein ${ }^{3}$, Benoit Baudry ${ }^{2}$, Yves le Traon ${ }^{1}$ \\ ${ }^{1}$ LASSY, University of Luxembourg, Luxembourg \{gilles.perrouin,yves.letraon\}@uni.lu \\ ${ }^{2}$ Triskell Team, IRISA/INRIA Rennes Bretagne Atlantique, $\{$ ssen,bbaudry $\} @$ irisa.fr \\ ${ }^{3}$ ISC Department, CRP Gabriel Lippmann, Luxembourg, klein@lippmann.lu
}

\begin{abstract}
Software Product Lines (SPL) are difficult to validate due to combinatorics induced by variability across their features. This leads to combinatorial explosion of the number of derivable products. Exhaustive testing in such a large space of products is infeasible. One possible option is to test SPLs by generating test cases that cover all possible $T$ feature interactions ( $T$-wise). $T$-wise dramatically reduces the number of test products while ensuring reasonable SPL coverage. However, automatic generation of test cases satisfying $T$-wise using SAT solvers raises two issues. The encoding of SPL models and $T$ wise criteria into a set of formulas acceptable by the solver and their satisfaction which fails when processed "all-at-once". We propose a scalable toolset using Alloy to automatically generate test cases satisfying $T$-wise from SPL models. We define strategies to split $T$-wise combinations into solvable subsets. We design and compute metrics to evaluate strategies on AspectOPTIMA, a concrete transactional SPL.

Index Terms-Model-based Engineering and Testing, Test Generation, T-wise and pairwise, Software Product Lines, Alloy
\end{abstract}

\section{INTRODUCTION}

When a company rapidly derives a wide range of different products a key-challenge is to ensure correctness and safety of most of these products (if not all) at a low cost. Software Product Line [1], [2] (SPL) techniques (and tools) allow engineering such families of related products. However, they rarely focus on testing the SPL as a whole. A software product line is usually modeled with a feature diagram [3], describing the set of features in the SPL and specifying the constraints and relationships between these features. For example, mandatory features as well as mutually exclusive ones can be described. As a result, from a feature diagram it is possible to derive products by selecting a set of features that satisfy all the constraints. The product is a software system built by composing the software assets that implement each feature.

Product line testing consists in deriving a set of products and in testing each product. This raises two major issues: 1) the explosion in the number possible products; 2) the generation of test suites for products. The first issue rises from the combinatorial growth in the number of products with the number of features. In realistic cases, the number of possible products is too large for exhaustive testing. Therefore, the challenge is to select a relevant subset of products for testing. The

This work was supported by the Luxembourg FNR SETER and SPLIT (FNR+CNRS) projects and by EU FP7 Grant agreement 215483 (S-Cube) second issue is to generate test inputs for testing each of the selected product. This can been seen as applying conventional testing techniques while exploiting the commonalities between products to reduce the testing effort [4], [5], [6]. Here, we focus on the first issue: How can we efficiently select a subset of products for product line testing?

Previous work [7], [8] has identified combinatorial interaction testing (CIT) as a relevant approach to reduce the number of products for testing. CIT is a systematic approach for sampling large domains of test data. It is based on the observation that most of the faults are triggered by interactions between a small numbers of variables. This has led to the definition of pairwise (or 2-wise) testing. This technique selects the set of all combinations so that all possible pairs of variable values are included in the set of test data. Pairwise testing has been generalized to $T$-wise testing which samples the input domain to cover all $T$-wise combinations. In the context of SPL testing, this consists of selecting the minimal set of products in which all $T$-wise feature interactions occur at least once.

Current algorithms for automatic generation of $T$-wise test data sets have a limited support in the presence of dependencies between variable values. This prevents the application of these algorithms in the context of software product lines since feature diagrams define complex dependencies between variables that cannot be ignored during product derivation. Previous work [9], [10] proposed to use constraints solvers as a possibility to deal with this issue. However, they still leave two open problems: scalability and the need for a formalism to express feature diagrams. The former is related to the limitations of constraint solvers when the number of variables and clauses increases. Above a certain limit, solvers cannot find a solution, which makes the approach infeasible in practice. The latter problem is related to the engineering of SPLs. Designers build feature diagrams using editors for a dedicated formalism. On the other hand, constraint solvers manipulate clauses, usually in Boolean Conjunctive Normal Form (CNF). Both formalisms are radically different in their expressiveness and modeling intention. This is a major barrier for the generation of $T$-wise configurations from feature diagrams.

In this paper, we propose an approach for scalable automatic generation of $T$-wise products from a feature diagram. Current constraint solvers have a limit in the number of clauses they can solve at once. It is necessary to divide the set of clauses 
into solvable subsets. We compose the solutions in the subsets to obtain a global set. In this work, we investigate two "divideand-compose" strategies to divide the problem of $T$-wise generation for a feature diagram into several sub problems that can be solved automatically. The solution to each sub problem is a set of products that cover some $T$-wise interactions. The union of these sets cover all interactions, thus satisfying the $T$-wise criterion on the feature diagram. However "divide-andcompose" strategies may yield a higher number of products to be tested and redundancy amongst them which is the price for scalability. We define metrics to compare the quality of these strategies and apply them on a concrete case study.

Our T-wise testing toolset first transforms a given feature diagram and its interactions into a set of constraints into Alloy [11], [12], a formal modeling language, based on firstorder logic, and suited for automatic instance generation. Then it complements the Alloy model with the definition of the $T$-wise criteria and applies one of the chosen strategies to produce a suite of products forming test cases. Finally, metrics are computed giving important information on the quality of the test suite. We extensively applied our toolset on AspectOPTIMA [13], [14] a concrete aspect-oriented SPL devoted to transactional management.

The remainder of the paper is structured as follows: Section II details the context of SPL testing and motivates the problem. Section III describes the metrics to assess SPL test generation strategies. Section IV gives an overview of the product generation approach. In Section V we present two "divide-andcompose" strategies. In Section VI we present experiments to qualify our strategies on the AspectOPTIMA SPL. Section VII presents related contributions in the field and Section VIII draws some conclusions and outlines future work.

\section{Context And Problem}

\section{A. Context}

In this paper, we focus on generating a small set of test products for a feature diagram. A product is a valid configuration of the feature diagram that can be used as a relevant test case for the SPL. We give a brief definition and example of feature diagrams before describing test case generation for such structures.

Feature Diagram: Feature Diagrams (FD) introduced by Kang et al. [3] compactly represent ( Figure 1) all the products of an SPL in terms of features ${ }^{1}$ which can be composed. Feature diagrams have been formalized to perform SPL analysis [15], [16], [17], [18]. In [16], [17], Schobbens et al. propose an generic formal definition of FD which subsumes many existing FD dialects. FDs are defined in terms of a parametric structure whose parameters serve to characterize each FD notation variant. GT (Graph Type) is a boolean parameter indicates whether the considered notation is a Direct Acyclic Graph (DAG) or a tree. NT (Node Type) is the set of boolean operators available for this FD notation. These

\footnotetext{
${ }^{1}$ Defined by Pamela Zave as "An increment in functionality". See http: //www.research.att.com/ pamela/faq.html
}

operators are of the form $o p_{k}$ with $k \in \mathbb{N}$ denoting the number of children nodes on which they apply to. Considered operators are and $_{k}$ (mandatory nodes), xor $_{k}$ (alternative nodes) or $_{k}$ (true if any of its child nodes is selected), opt $k_{k}$ (optional nodes). Finally $v p(i . . j)_{k}(i \in \mathbb{N}$ and $j \in \mathbb{N} \cup *)$ is true if at least $i$ and at most $j$ of its $\mathrm{k}$ nodes are selected. Existing other boolean operators can usually be expressed with $v p$. $G C T$ (Graphical Constraint Type) is the set of binary boolean functions that can be expressed graphically. A typical example is the "requires" between two features. Finally, TCL (Textual Constraint Language) tells if and how we can specify boolean constraints amongst nodes. A FD is defined as follows:

- A set of nodes $N$, which is further decomposed into a set of primitive nodes $P$ (which have a direct interest for the product). Other nodes are used for decomposition purposes. A special root node, $r$ represents the top of the decomposition,

- A function $\lambda: N \mapsto N T$ that labels each node with a boolean operator,

- A set $D E \in N \times N$ of decomposition edges. As FDs are directed, node $n 1, n 2 \in N,(n 1, n 2) \in D E$ will be noted $n 1 \rightarrow n 2$ where $\mathrm{n} 1$ is the parent and $\mathrm{n} 2$ the child,

- A set $C E \in N \times G C T \times N$ of constraint edges,

- A set $\phi \in T C L$

A FD has also some well-formedness rules to be valid: only root $(r)$ has no parent; a FD is acyclic; if GT = true the graph is a tree; the arity of boolean operators must be respected. We build upon this formalization to create feature modeling environments supporting product derivation [19] where we encode the AspectOPTIMA SPL feature diagram (see figure 1). We implement AspectOptima SPL as an aspectoriented framework providing run-time support for different transaction models. AspectOptima has been proposed in [14], [13] as an independent case study to evaluate aspect-oriented software development approaches, in particular aspect-oriented modeling techniques. Once we defined the FD, we can create products (i.e a selection of features in the FD). To be valid, a product follows these rules: 1) The root feature has to be in the selection, 2) The selection should evaluate to true for all operators referencing them, 3) All constraints (graphical and textual) must be satisfied 4) For any feature that is not the root, its parent(s) have to be in the selection. We enforce the validity of a product according to well-formedness rules defined on our generic metamodel [19] which are automatically translated to Alloy by our FeatureDiagram2Alloy transformation (see Section IV). Once we introduce the notion of feature diagram and formalize it we can form our notion of SPL testing on such an entity.

SPL Test Case: A SPL test case is one valid product (i.e. a ) of the product line. Once this test case is generated from a feature diagram, its behaviour has to be tested.

SPL Test Suite: A SPL Test Suite is a set of SPL test cases.

Example: Figure 2 presents 3 test cases, three products which can be derived from the feature model. These three test cases form a test suite. 


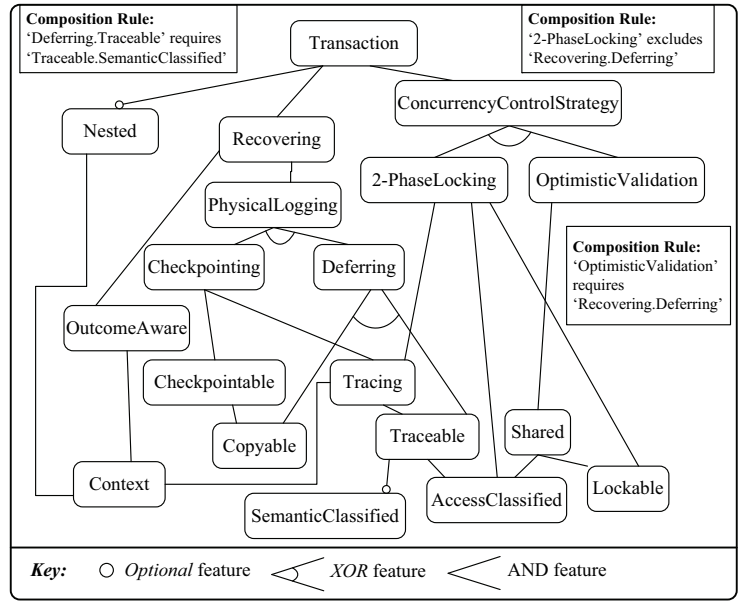

Fig. 1. Feature Diagram of AspectOptimA

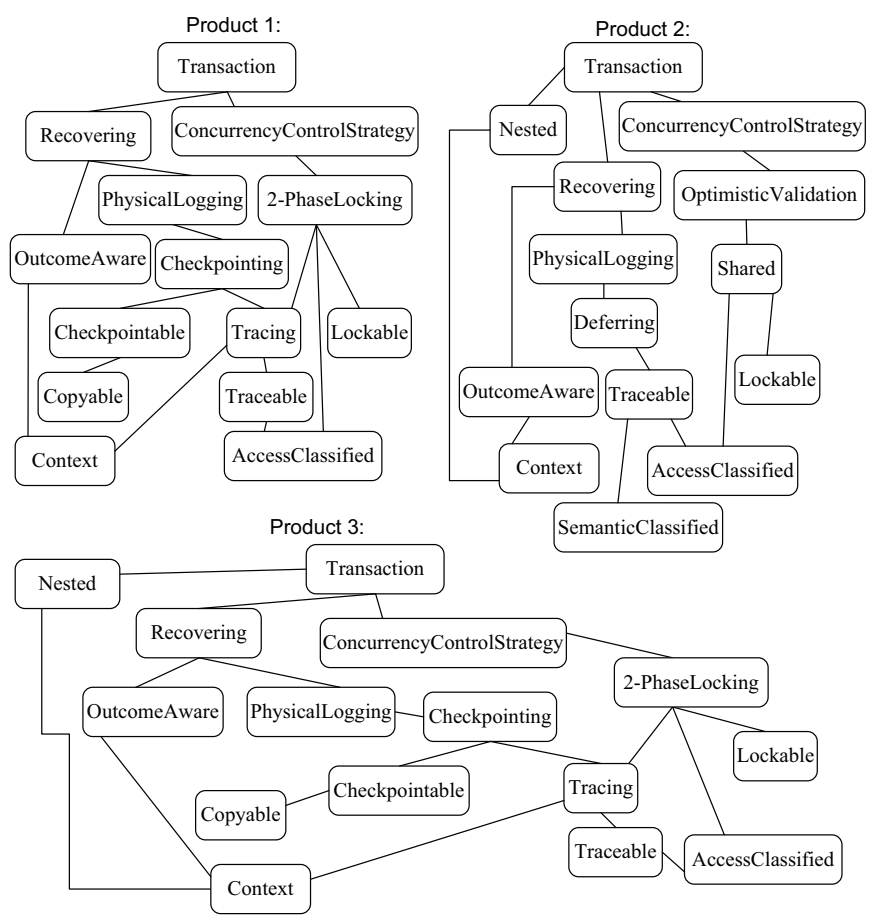

Fig. 2. Three Test Cases

Valid/Invalid T-tuple: A $T$-tuple ( were $T$ is a natural integer giving the number of features present in the $T$ tuple $^{2}$ ) of features is said to be valid (respectively invalid), if it is possible (respectively impossible) to derive a product that contains the pair ( $T$-tuple) while satisfying the feature diagram's constraints.

Example: In the AspectOptima product line we have a total of 19 features. All these 19 features can take the value true or false. Thus, we can generate 681 pairs that

\footnotetext{
${ }^{2}$ In general we will use the term "tuple" to mention a $T$-tuple when $t$ does not matter. In the special case of pairwise, i.e. when $T=2$, we denote a 2-tuple by the term "pair".
}

cover all pairwise combinations of feature values. However, not all of these pairs can be part of a product derivable from the feature model. For example, the pair $<$ (not Transaction), Recovering $>$ is invalid with respect to the AspectOptima feature diagram which specifies that the feature Transaction is mandatory.

SPL test adequacy criterion: To determine whether a test suite is able to cover the feature model of the SPL, we need to express test adequacy conditions. In particular, we consider the "T-wise" [8], [9] adequacy criterion (all- $T$-tuples) were each valid $T$-tuple of features is required to appear in at least one test case.

Example: The test suite presented in figure 2 does not satisfy our adequacy criterion since the pair (2-tuple) <semantic classified, lockable> does not appear in any of the three test cases.

Test generation: In our context of SPL testing, test generation consists of analyzing a feature diagram in order to generate a test suite that satisfies pairwise coverage.

Pairwise (and more generally T-wise) is a set of constraints over a range of variables (mathematically defined as covering arrays [20]). Thus it is possible to use SAT-solving technology [21], [22], [23] to compute such arrays. In our case, variables are the features of a given given feature diagram. It is therefore mandatory to encode a feature diagram in first order logic so that SAT-solvers can analyze them. Thanks to feature diagram formalization, this is possible [15], [18] and have been done for various purposes [24], [25].

\section{B. Problem}

The work in this paper builds upon this idea: model the test generation problem as a set of constraints and ask a constraint solver for solutions. In this context we tackle two issues: (1) modelling the SPL test generation problem in order to use a constraint solver and (2) dealing with the scalability limitations of SAT solvers. Our contribution on the first issue is an automatic transformation from a feature diagram to an Alloy [12] model.

Scalability is a major issue with SAT solvers. It is known that solving a SAT formula on more than 2 variables is an NP-complete problem. It is also known that depending on the number of variables and the number of clauses, satisfiability or unsatisfiability is more or less computationally complex [26]. However, we currently don't know how to predict the computation complexity of a given problem. An empirical approach thus consists in trying to solve the set of "constraints all-at-once". Three things can happen: the solver returns a solution, the solver returns an unsatisfiability verdict, the solver crashes because the problem is too complex. In the latter case, one way to generate a test suite that covers twise interactions, is to decompose the problem into simpler problems, solve them independently and merge the solutions. In the following, we refer to this approach as "divide-andcompose" approach.

One pragmatic approach, and a naive one, consists of running the solver once for each $T$-tuple that has to be covered. 
This iterative process is the simplest "divide-and-compose" approach and it generates one test case for each valid $T$ tuple in the FD. For the AspectOptima SPL, we obtain 421 test cases that satisfy pairwise and that corresponds to 421 products to be tested. The all-pairs criterion is satisfied but with a large number of products. It also has to be noted that only 128 different products can be instantiated from the AspectOptima SPL. This indicates that the application of "divide-and-compose", although it might define problems that can be solved, also introduces a large number of redundant test cases in the resulting test suite. Indeed, if it generates 421 test cases, but there can be only 128 different test cases, there is an important redundancy rate.

In general, a solution for generating a test suite with a SAT solver consists in finding a strategy to decompose the SAT problem in smaller problems that can be automatically solved. Also, the strategy should decompose the problem in such a way that when the solutions to all sub-problems are composed, the amount of redundancy in the suite is limited.

Test generation strategies: In this paper, we call strategies the ways we "divide-and-compose". Depending on applied strategies and their parameters we will derive more or less test cases. Before delving into the two different strategies we will introduce metrics to evaluate them in the next section.

\section{Metrics for Strategy Evaluation}

We need efficiency and quality attributes in order to evaluate the generated SPL test cases and compare the automatic generation strategies. The first efficiency attribute relates to the size of the generated SPL test suite.

SPL Test suite size: The size of a test suite is defined by the number of SPL test cases that a given generation strategy computes. In the best case, we want a strategy to generate the minimal number of test cases to satisfy the SPL test adequacy criterion. As this optimal number is generally not known a priori, we use the SPL test suite size as a relative measure to compare test generation strategies.

A second efficiency attribute relates to the cost of test generation in itself. We measure this cost as the time taken for generation.

SPL strategy time taken: We characterize the cost of a given strategy by the time it took to decompose the problem into solvable sub-problems and the time it took to merge the partial generated solutions to a SPL test suite.

We also evaluate the quality of the generated test cases. First, we want to appreciate the coverage of the generated test cases with respect to the feature diagram. We measure coverage by looking at the rate of similarity between the test cases that are generated. The intuition is that, the more test cases are similar, the less they cover the variety of products that can be generated from the feature diagram.

1) Test Case Redundancy: We define test case redundancy between two valid products as the ratio of non-compulsory features they have in common. By compulsory, we mean that it comprises mandatory features and features that are explicitly required by them. Put in other terms, for any set of features $F \subseteq N$ representing a valid product according to the aforementioned rules for constructing FDs in section II, we form the set $C F \subseteq F$ :

$$
\begin{aligned}
C F=\left\{\left\{f_{i}\right\} \in N \mid \forall\left\{f_{j}\right\} \in N \wedge f_{j} \mapsto f_{i},\right. \\
\forall k \in \mathbb{N}, \lambda\left(f_{j}\right)=\operatorname{and}_{k} \cup \\
\quad\left\{f_{l}\right\} \in N \mid \text { requires }\left(f_{i}, f_{l}\right)=\text { true }
\end{aligned}
$$

In which requires is a binary boolean function (belonging to $G C T$ ) such that it returns true if there is a constraint edge labeled as "requires" between theses two features.

Hence the redundancy ratio between two test products is:

$$
r\left(p_{i}, p_{j}\right)=\frac{\operatorname{card}\left(\left(F_{p_{i}}-C F_{p_{i}}\right) \cap\left(F_{p_{j}}-C F_{p_{j}}\right)\right)}{\operatorname{card}\left(\left(F_{p_{i}}-C F_{p_{i}}\right) \cup\left(F_{p_{j}}-C F_{p_{j}}\right)\right)}
$$

This ratio equals to 1 if the two products are the same and 0 if they have no non-compulsory feature in common.

Example: Products 1 and 3 (Figure 2) have test case ratio of 0.88 since they differ only by one feature out of 9 noncompulsory.

At the test suite level, we compute test case redundancy by computing the average of test case redundancy ratio for any two (cartesian product) test cases of the suite.

As a second quality attribute, we want to assess the quality of the generated SPL test cases with respect to $T$-wise interactions coverage. If we know that, by construction, each tuple appears at least once in the test suite, we also know that the generation process might lead to the repetition of tuples an arbitrary number of times. For the SPL testers, such repetitions imply that they will test the same interaction of features several times.

T-tuple Occurrence: is the number of occurrences of a valid pair ( $T$-tuple) in a test suite. Let $T S$ a test suite comprised of $p_{i}$ valid cases and $F_{p_{i}} \subseteq N$ their associated features. Let $T$ a $T$-tuple ( $T=\left\{f_{i} \in N\right\}$ ). Tuple redundancy is then:

$$
t_{o}=\operatorname{card}\left(t \in T \mid t \subseteq F_{p_{i}}\right)
$$

\section{Test Generation Methodology \& Toolset}

In this section, we describe the automatic generation of test products from a feature diagram that satisfy the $T$ wise SPL test adequacy criteria. Our tool support has been designed to support any value of $T$. The toolset has been implemented mostly in JAVA (approximatively $2.3 \mathrm{KLOC}$ ) for T-wise generation and metrics computation and Kermeta [27] for transforming feature diagrams into alloy specifications. The methodology consists of five key steps shown in Figure 3. The generation is based on Alloy as the underlying formalism to formally capture all dependencies between features in a feature diagram as well as the the interactions that should be covered by the test cases. Alloy is a formalism dedicated to lightweight formal analysis [12]. Alloy provides a set of concepts allowing to specify elements and constraints between them. The first construct is Signature (sig). A signature defines a set of elements and possibly the relationships with other elements. Signatures are similar to type declarations in an object-oriented language. Facts (fact) are axioms that specify constraints 


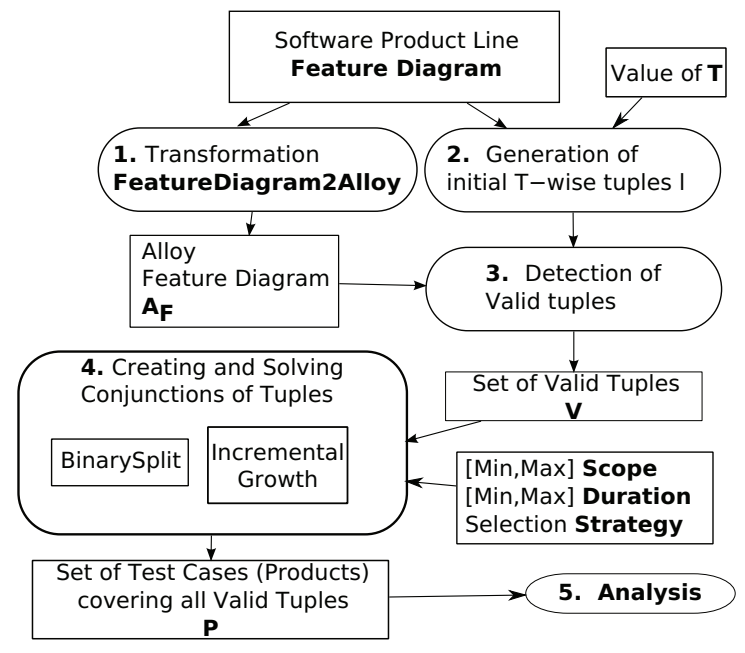

Fig. 3. Product Line Test Generation Methodology

about elements and relationships. These axioms must always hold, they are close to the concept of invariants in other specification languages. Predicates, (pred), as opposed to facts, define constraints which can evaluate to true or false. With these constructs it is possible to construct various kinds of Alloy models and to ask alloy if it is possible to find instances that satisfy all constraints and evaluate one predicate to true. The scope is an integer bound on the maximum number of instances for each signature [12]. This allows to limit the search space in which Alloy looks for a solutions and this is a way to finely tune how Alloy builds instances satisfying a model.

\section{A. Step 1: Transforming Feature Diagrams to Alloy}

In order to generate valid test products directly from a feature diagram, we need to transform the diagram in a model that captures constraints between features (defined in Section II). The FeatureDiagram2Alloy transformation automatically generates an Alloy model $A_{F}$ from any feature diagram $F D$ expressed in our generic feature diagram formalism [19].

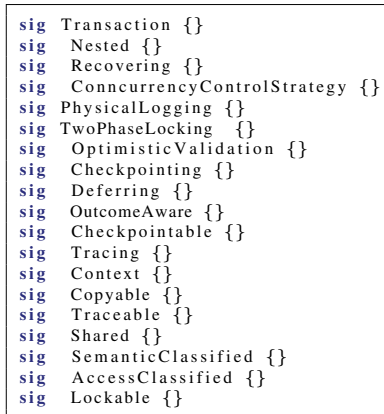

Listing 1. Generated Signatures for Features in AspectOptima

The $A_{F}$ model captures all features as Alloy signatures and a set of Alloy signatures that capture all constraints and relationships between features. This model also declares two signatures that are specific to test generation: configuration that corresponds to a test case and that encapsulates a set of features (listing 2); ProductConfiguration (listing 3) which will encapsulate a set of test cases.

Example: In the AspectOptima feature diagram, shown in Figure 1 , we have 19 features $f_{1}, f_{2}, \ldots, f_{19}$. The transformation FeatureDiagram2Alloy generates 19 signatures to represent these features shown in listing 1 .

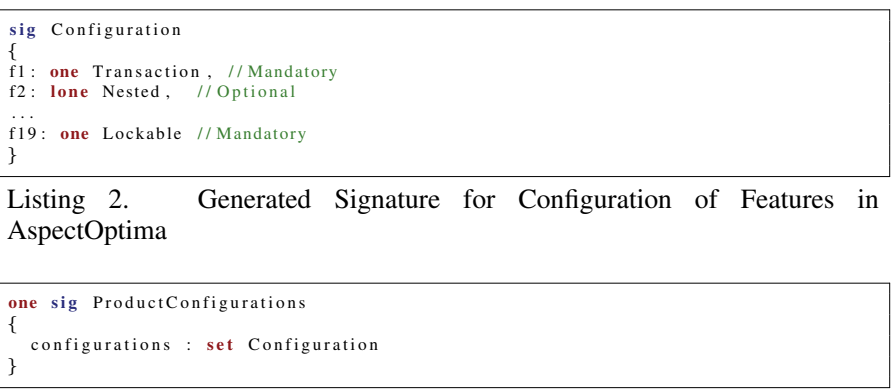

Listing 3. Generated Signature for Set of Configurations

The FeatureDiagram2Alloy transformation generates Alloy facts in $A_{F}$.

Example: In the listing 4 we present two generated Alloy facts showing mutually exclusive (XOR) features selection: $f_{6}$ (TwoPhaseLocking) and $f_{7}$ (OptimisticValidation) given that $f_{4}$ (ConcurrencyControlStrategy) has been selected. These facts must be true for all configurations.

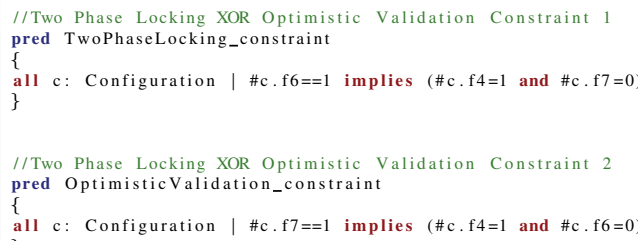

Listing 4. Generated Fact for XOR

The FeatureDiagram2Alloy transformation has been implemented as a model transformation in the Kermeta metamodeling environement [27]. Since our feature diagram formalism is generic [19] various kinds of feature diagrams can be automatically transformed.

\section{B. Step 2: Generation of Tuples}

In Step 2, we automatically compute the set $I$ of all possible tuples from feature diagram $A F$ and the number $T$. The tuples enumerate all $T$-wise interactions between all selections of features in $A F$.

Example: The 3-tuple $t=<\# f_{1}=0, \# f_{2}=1, \# f_{3}=$ $1>$ for the value $T=3$ contains 3 features and their valuations. In the tuple we state that the set of test products must contain at least one test case that has features $f_{2}$ and $f_{3}$ and does not have $\mathrm{f} 1$.

The initial set of tuples $I$ is the set of tuples that cover all combinations of $T$ features taken at a time. For example, if there are $N$ features then the size of $I$ is ${ }_{2 N} C_{T}$ minus all tuples with repetitions of the same selected feature.

Each tuple $t$ in $I$ also has an Alloy predicate representation. An Alloy predicate representation of a tuple $t$ is t.predicate. 
Example: The tuple $t=<\# f_{1}=0, \# f_{2}=1, \# f_{3}=1>$ is shown in listing 5 .

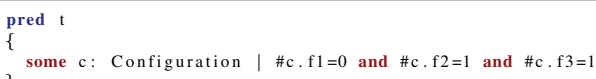

Listing 5. Example Tuple Predicate

\section{Step 3: Detection of Valid Tuples}

In this third step, we use the predicates derived from each possible tuple in order to select the valid ones according to the feature diagram. We say that a tuple is valid if it can be present in a valid instance of the feature diagram $F$.

Example: Consider AspectOptima (in Figure 1) features $f_{1}$ :Transaction, $f_{2}$ :Nested, and $f_{4}$ :ConcurrencyControlStrategy, The 3-tuple $t=<\# f_{1}=$ $0, \# f_{2}=1, \# f_{4}=1>$ is not a valid tuple as the feature $f_{4}$ required the existence of feature $f_{1}$ and hence we neglect it. On the other hand, the 3-tuple $t=<\# f_{1}=1, \# f_{2}=0, \# f_{4}=1>$ is valid since all feature selections hold true for $F$. We determine the validity of each such tuple $t$ by solving $A_{F} \cup t$.predicate for a scope of exactly 1. This translates to solving the Alloy model to obtain exactly one product for which the tuple $t$ holds true.

Example: For the AspectOptima case study we generate 681 tuples for pair-wise $(T=2)$ interactions in the initial set $I$. We select 421 valid tuples in the set $V$.

\section{Step 4: Creating and Solving Conjunctions of Multiple Tuples}

Once we have a set of valid tuples, we can start generating a test suite according to the $T$-wise SPL adequacy criteria. Intuitively, this consists in combining all valid tuples from $V$ with respect to $A_{F}$ in order to generate test products that cover all T-wise interactions.

Example: For pair-wise testing in the case of AspectOptima this amounts to solving a conjunction of 421 tuple predicates $t_{1}$.predicate $\cap t_{2}$.predicate $\cap \ldots \cap t_{421}$.predicate for a certain scope.

The major issue we tackle in this work is that in general, constraint solvers cannot generate the conjunction of all valid tuples at once.

Example: Using the "all-at-once" strategy on aspectOPTIMA, with 421 valid tuples, the generation process crashes without giving any solution after several minutes using MiniSAT [23] solver.

Hence we derived two "divide-and-compose" strategies to break down the problem of solving a conjunction of tuples to smaller subsets of conjunction of tuples. The strategies we present are Binary Split and Incremental Growth. Each strategy is parameterized by intervals of values defining the scope of research for each (sub)-conjunction of tuples, the duration in which Alloy is authorized to solve the conjunction as well as a strategy defining how features are picked in a tuple. We describe these strategies in more detail in section $\mathrm{V}$. The combination of solutions is a test suite $T S$ that covers all tuples.
1) Step 5: Analysis: In order to assess the suitability of our "divide-and-compose" strategies and compare their ability to generate test suites, we need to compute the metrics defined in section III. We compute for each generated test suite the number of products or test cases, test case and tuple redundancy. We performed extensive experimentation on AspectOPTIMA by generating test suite with different scope and time values. We present consolidated results of these experiments in section VI.

\section{Two strategies for $T$-Wise SPL Test Suite GENERATION}

As mentioned previously, to be scalable we divide the problem of solving tuples into sub-problems, i.e. we are creating conjunctions of subsets of tuples. We solve the conjunction of tuples in each of these subsets using the algorithm presented in Section V-A. The first strategy to obtain subsets of tuples, Binary Split, is discussed in Section V-B. We present the second strategy, Incremental Growth, in Section V-C.

\section{A. Solving a Conjunction of Tuples}

We solve a conjunction of tuples using the Algorithm 1. We combine the Alloy model $A_{F}$ with a predicate $C T(S)$.predicate representing the conjunction of tuples in the set $S=t_{1}, t_{2}, \ldots, t_{L}$. We solve the resulting Alloy model $m$ using incremental scoping. We create a run command $c$ starting for a scope between the minimum scope $m n S c$ and the max scope $m x S$ cope. We insert the command $c$ into $m$. A SAT solver such as MiniSAT [23] or ZChaff [22] is used to solve $m$. We determine the duration dur = startTime-endTime for each scope value. If $d u r$ exceeds maximum duration mxDur we stop incrementing the scope. The solve method returns the result of the SAT solving and the corresponding solution if a solution exists.

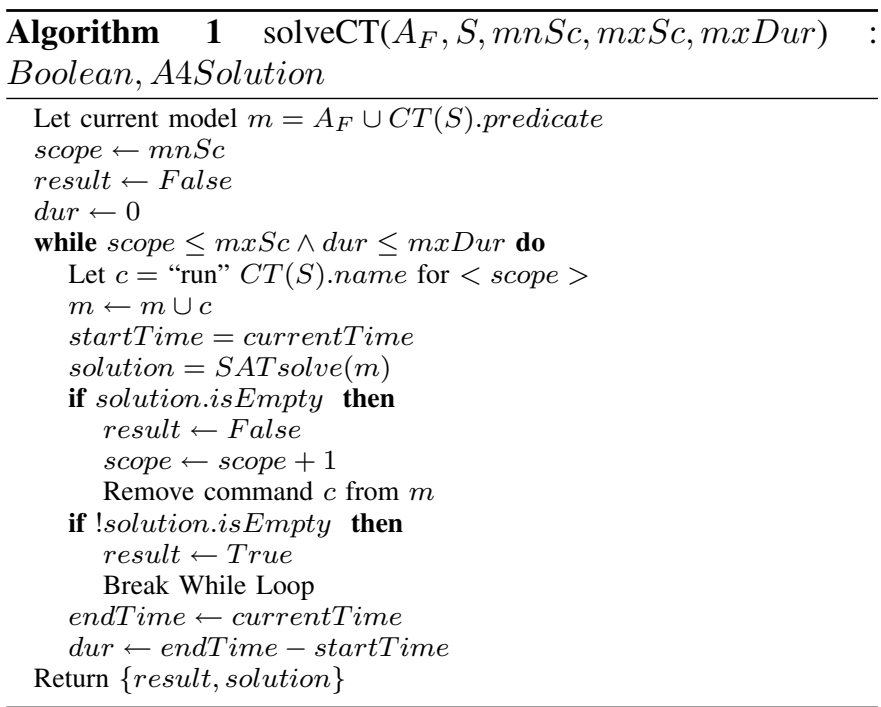

\section{B. Binary Split}

The binary split algorithm shown in Algorithm 2 is based on splitting the set of all valid tuples $V$ into subsets (halves) 
until all subsets of tuples are solvable. We first order the set of valid tuples based on the strategy Str. The strategy can be random or based on distance measure. In this paper, we consider a random ordering. The Pool is set of sets of tuples. Initially, Pool contains the entire set of valid tuples $V$. If each set of tuples Pool[i],0 $\leq i \leq$ Pool.size in Pool is not solvable in the given range of scopes $m n S c$ and $m x S c$ or within the maximum duration $m x D$ ur then result is False for Pool $[i]$. A single value of result $=$ False renders AllResult $=$ False. In such a case, we select the largest set in Pool $[i]$ and split it into halves $\{H 1\}$ and $\{H 2\}$. We insert the halves $\{H 1\}$ and $\{H 2\}$ into Pool $[i]$. The process is repeated until all sets of tuples in Pool can be solved given the time limits and AllResult = True. In the worst case, binary split convergences with one tuple a set making Pool.size $=$ V.size as all tuples in $V$ are solvable.

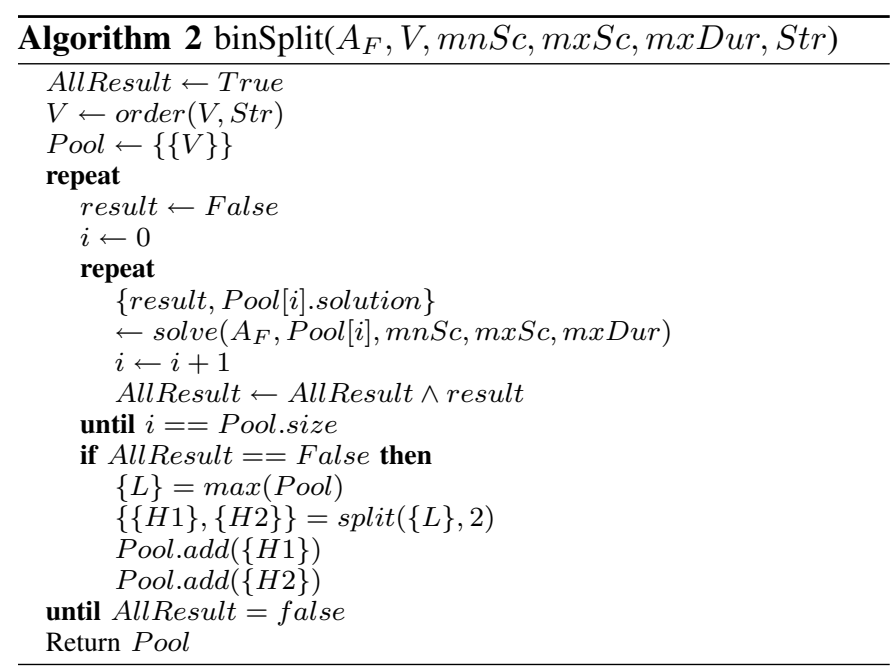

\section{Incremental Growth}

The incremental growth is shown in Algorithm 3. In the algorithm we incrementally build a set of tuples in the conjunction $C T$ and add it to the Pool. The select function based on a strategy Str selects a tuple in $V$ and inserts it into $C T$. The strategy $S t r$ can be random or based on a distance measure between tuples. In this paper, we consider only a random strategy for selection. We select and remove a tuple from $V$ and add it to $C T$ until the conjunction cannot be solved anymore ,i.e. result $=$ False. We remove the last tuple and put it back into $V$. We include $C T$ into Pool. In every iteration, we initialize a new conjunction of tuples until we obtain sets of tuples in Pool that contain all tuples initially in $V$ or when $V$ is empty.

\section{EXPERIMENTS}

The objective for our experiments is: To demonstrate the feasibility of "divide-and-compose" strategies (Binary Split and Incremental Growth) and compare their efficiency with respect to test case generation. All experiments are performed on a real-life feature model: AspectOPTIMA. In this section

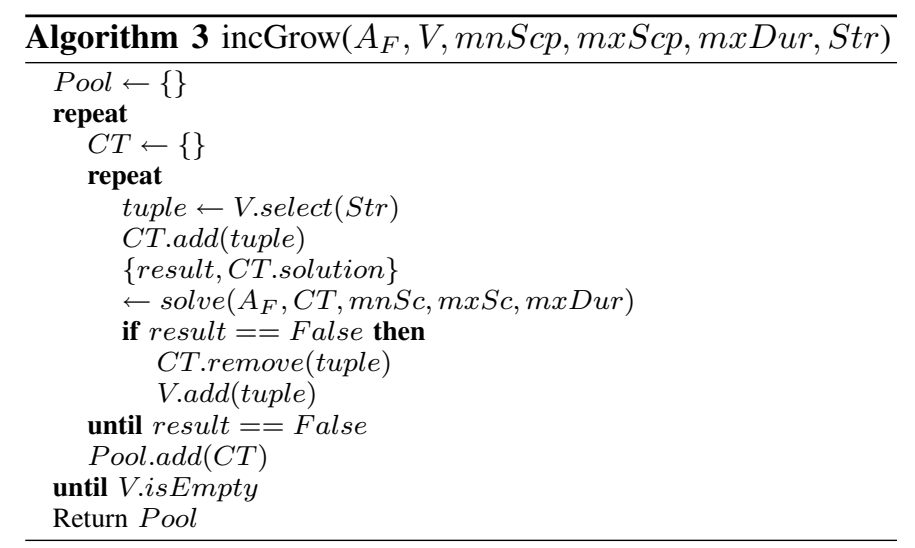

we report and discuss the automatic generation of T-wise test suites for this model.

\section{A. Experimental Setting}

We automatically generate test suites with the two "divideand-compose" strategies and compare them according to: (a) the number of generated test cases; (b) the number of tuple occurrences in the test suites; (c) the similarity of the products in the generated test suites. For both strategies we have to set the values for two parameters that specify the search space: the scope and the time limit. We vary the scope over 5 values: $3,4,5,6,7$; the maximum duration $m x D u r$ to find a solution for a given conjunction of constraints is fixed at $1600 \mathrm{~ms}$. We generate 100 sets of products for each scope giving us a total of $5 \times 100$ sets of products for a strategy. The reason we generate 100 solutions is to study the variability in the solutions given that we use uniform random ordering in binary split and random tuple selection in incremental growth. Therefore, for two strategies we have $2 \times 5 \times 100$ sets of products or test cases. We perform our experiments on a MacBook Pro 2007 laptop with the Intel Core 2 Duo processor and 2GB of RAM.

Before studying the results of our experiments we note that attempting "solving-all-constraints-as-once" does not yield any solutions for the AspectOPTIMA SPL. This is true even for simple feature models such as AspectOPTIMA that does not lead to derivation of billions of products (like industrial product lines). On the other hand, all executions of both "divide-and-compose" strategies generate T-wise test suites. This first observation indicates that these strategies enable the usage of SAT solvers for the automatic generation of T-wise interactions test suites for both simple and potentially complex feature models. This is the first main result of our study.

\section{B. Number of Products Vs. Scope}

In Figure 4, we present the number of products generated for different scopes, which corresponds to the number of test cases in a suite. Each box and its whiskers correspond to 100 solutions generated using a strategy for a given scope. On the $\mathrm{x}$-axis we have scope for two strategies: BinarySplit represented by bin_scope and IncrementalGrowth represented by inc_scope. 


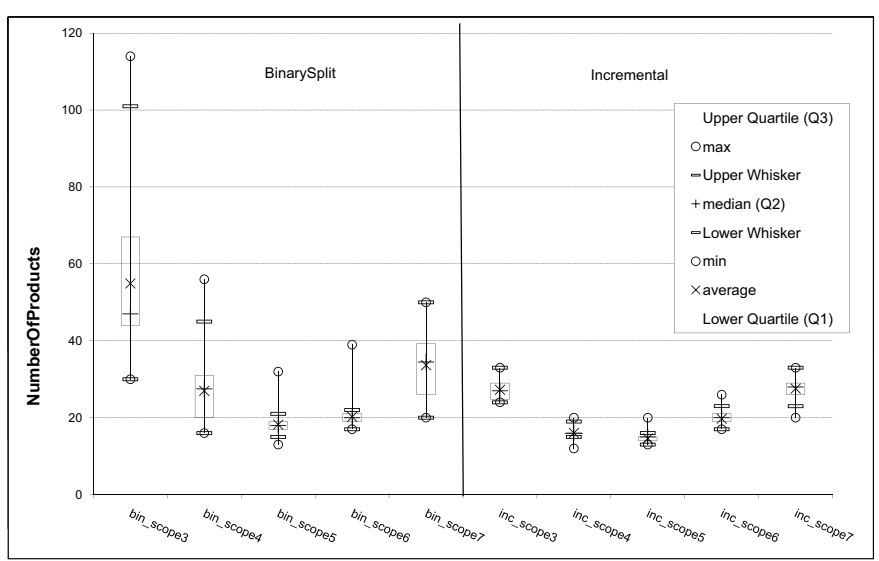

Fig. 4. Box Plot for Number of Products vs. Scope

For the binary split strategy, the number of products is high for a scope of 3 (average of 50 products), decreases towards a scope of 5 (average 18 products) and increases again towards a scope of 7 (average of 35 products). In our experiments the scope nearest to the minimal number of test cases is 5 . For a scope of 7 we ask the solver to create 7 products per subset of tuples (or pairs) while only 5 products suffice for the same set of tuples leading to more products that satisfy the same set of tuples. This is true for highly constrained SPLs such as AspectOPTIMA where the total number of products generated does not exceed a couple of hundred. Therefore, fewer products are sufficient to capture all T-wise interactions. For a scope too small such as 3 , binary split gives a large number of products. This comes from the coarse-grain splitting (into halves) of the set of tuples leading to the non-optimal use of 3 products to cover a maximum number of tuples.

For the incremental growth, the general trend that is the high number of products for a scope of 3 (average 25 products), decrease towards a scope of 5 (average 17 products), and increase again towards a scope of 7 (average 27 products). The reasoning for this general trend is similar to binary splitting except that incremental growth attempts to optimize the number of tuples that can be squeezed into a product.

When comparing binary split and incremental growth, there is a notable difference in the variability in the solutions. Binary split results in a large variability (minimum 18 products at scope 5 to a maximum of 115 products at scope 3 ) in the number of products compared to incremental growth (minimum 16 products to a maximum of 30 products). This is reasonable as binary split applies a coarse-grain strategy of halving sets while incremental growth applies a selective strategy to 'squeeze in' a maximum number of tuples into a test suite. However, in terms of performance binary split for the AspectOPTIMA case study is far superior compared to incremental growth. Binary split takes an average of $641 \mathrm{~ms}$ to obtain a set of products for a scope of 3 while incremental growth takes about $14000 \mathrm{~ms}$. This is primarily due to the fewer steps (average 20) to divide in binary split compared to large number of steps (average 420) for incremental growth.
Therefore, we have a trade-off between the size of the test suite and the time to generate the suite. Both strategies are able to automatically find a small number of test cases satisfying all valid pairs of feature interactions.

\section{Tuple Occurrence Vs. Scope}

In Figure 5, we present a box plot showing the total occurrence of tuples for different scopes. We know that a possible limitation of divide-and-compose strategies is that they can generate test cases that cover the same tuple multiple times. This is a limitation for the testing effort, since a redundant tuple means that the same interaction of features has to be tested several times. The total number of valid tuples is 421 for AspectOPTIMA and hence ideally we would like to have a minimum number of products with exactly one occurrence of a tuple. However, the existence of mandatory features force to have multiple occurrences of some tuples in the suite. An effective strategy for test generation is thus a strategy that limits the occurrence of the same tuple in the test suite.

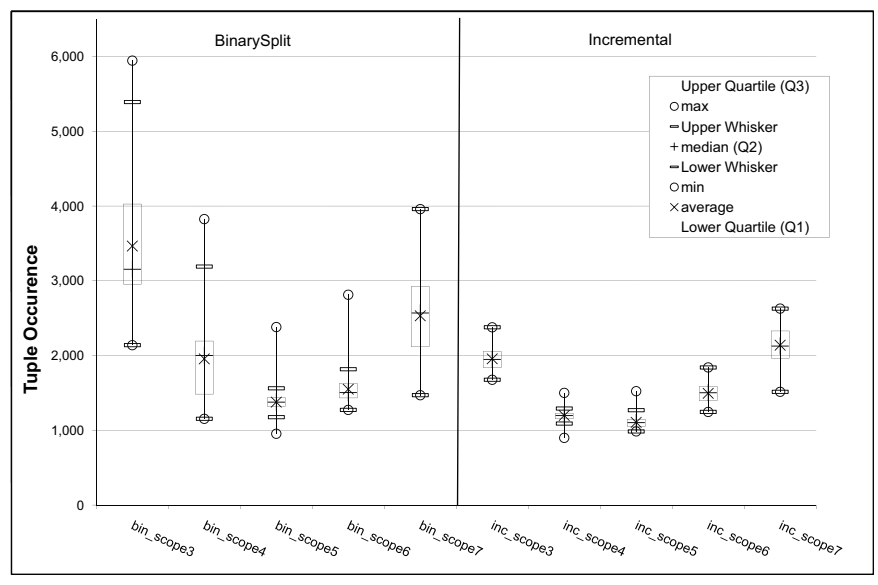

Fig. 5. Box Plot for Total Tuple Occurrence vs. Scope

For binary split, the total tuple occurrence for a scope of 3 is about 3000 on an average, decreases to about 1400 for a scope of 5 and increases again to 2500 for a scope of 7 . Therefore, a scope of 3 generates products with about 7 times the total tuple occurrence compared to the ideal unique occurrence, scope of 5 about 3 times. We again observe that the near-optimal scope of 5 has the least total tuple repetition.

For incremental growth, the total tuple occurrences are lower compared to binary split. Binary split and scope 3 gives products with 1.6 times more occurrences compared to incremental growth. In general, incremental growth converges to a better set of products: less products with less occurrences of tuples. The strategy and the scope help us choose the ideal set of test cases.

\section{Test Case Redundancy}

Results for test case redundancy are presented in Figure 6. One first observation is that the values are similar (except for scope 3) for BinarySplit and IncrementalGrowth strategies. This can be because both strategies are based on random 
ordering of tuples. Hence the coverage of the feature diagram by SPL test cases is quite similar and its particular structure does not influence test case redundancy between the two strategies.

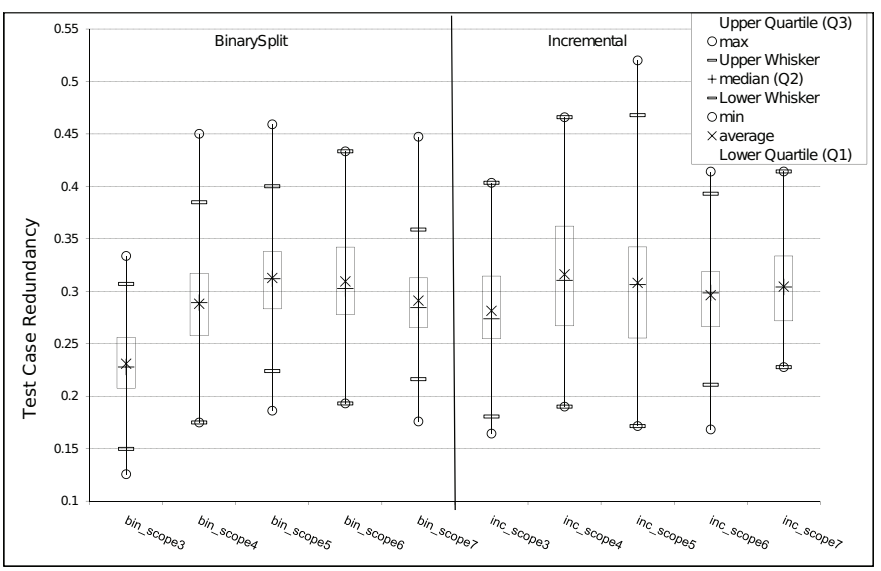

Fig. 6. Box Plot for Test Case Redundancy

We also observe that test case redundancy increases when the number of products decreases for both strategies, the minimum being obtained with scope 5 . This can be explained by the fact that when the number of products decreases, the generator must "fill" each product with more non-compulsory features in order to cover each tuple at least once. When we give more "freedom" to the strategies (by increasing the number of products), they have more options to fill products with non-compulsory features and generate less test case redundancy on average. High redundancy in a small test suite can be beneficial for test cases reuse [4]. However, high redundancy also means similar test cases in a suite and thus less coverage of the SPL, which might not be a good caracteristic of a test suite.

\section{E. Threats to Validity}

This work mainly focused on the definition of two divideand-compose strategies and the experiment was performed on only one real-world feature diagram. It is a realistic FD, in size and complexity of the constraints between feature. However, since we evaluate our strategies only on this one, there is an important threat to external validity. We cannot know how the trends we observed for both strategies can be generalized to feature diagrams with more features or a different topology. We are currently running similar experiments on larger feature models (and less constrained) to assess the impact of topology on the effectiveness of our strategies and implementation. We also have another threat to construct validity: we have developed the tools to measure the different metrics on the test suites. Concerning the metrics themselves, they are usual metrics to evaluate test suites (number of test cases, coverage) that we believe are relevant for the evaluation of the proposed strategies.

\section{RELATED WORK}

Our work deals with software-engineering specific dimensions of SPL testing: (1) scalability of test cases generation, (2) reduction of the resulting test cases set (both in terms of size of the test suite and redundancies) and (3) usability for the testers.

Concerning test generation for PL (1), McGregor [6] and Tevanlinna [5] propose a well-structured overview of the main challenges for testing product lines. A major one is obviously the exponential growth of possible products. The idea of using combinatorial testing for PL test selection is not new and has been initially proposed by Cohen et. al. [9], [10]. Combinatorial interaction testing (CIT) [7]. [8] led to the definition of pairwise testing, and then its generalization to twise testing. Cohen et. al. have applied CIT to systematically select configurations/products [9] that should be tested. They consider various algorithms in order to compute configurations that satisfy pair-wise and T-wise criteria [10]. Our work goes along the same lines but deals with scalability of the test generation, noting that CIT+SAT approaches do not scale directly with real-case feature diagrams, such as the AspectOPTIMA SPL example.

Concerning test minimization for PL (2), to limit repeated testing efforts, a possible solution is to produce template system test cases, common to the whole product line and that can be adapted to each product. Nebut et al. [28] proposed a model-based approach to derive test objectives for the whole system. In [29], Scheidemann defined a method minimizing the set of configurations to verify the whole software product line. The author exploits the commonalities in order to minimize the verification effort required for requirements that pertain to several configurations. However, this approach does not take into account constraints between features which limits the applicability of the approach (see [10]). In the same vein, [30] propose a method to generate test plans covering user-specified portions of the huge number of possible configurations of a component-based software system.

Concerning the last point (3), we choose a model driven technique to automatically map a feature diagram into an Alloy input format. The user of the approach can thus manipulate directly feature diagrams and transform them directly in Alloy. A formalization for feature models in Alloy can be found in [31], but is not dedicated to testing and feature diagrams have to be written by hand. Uzuncoava et al. [4] use Alloy to generate a test suite incrementally from the specification of a product, directly modeled as alloy formulae. The interesting point in this work is that tests are reused from one product to another in a cumulative way. Our work focuses on testing the SPL as whole rather than individual products. Indeed, these techniques of SPL testing are complementary, our method focusing on automated selection of products, which can then be individually tested.

Usability is also a question of analysis algorithms and case tools to manipulate and reason about feature models [24], [32]. Benavides et al. have developed FAMA [33] a generic 
open-source framework supporting various kinds of analyses. Minimal test-set computation is not part of them but our EMF/Eclipse based T-wise toolset can be integrated easily to it. Furthermore, our variability metamodel is generic and has been successfully applied/reused for product line derivation [19].

\section{CONCLUSION}

In this paper, we proposed an approach and platform supporting the automated generation of test cases for software product lines. Our work is motivated by concerns of scalability and usability. With respect to the first concern, we combined combinatorial interaction testing, as a systematic way to sample a small set of test cases, with two "divide-andcompose" strategies. These strategies address the scalability limitations of SAT solvers used to generate test cases that satisfy all constraints captured in a feature model. Using these strategies, we are able to automatically generate sets of test cases for a medium-sized realistic SPL such as AspectopTIMA which could not be processed in an "all-constraints-at-once" fashion. We assessed our strategies by computing metrics and discussed the factors that influence test case generation. We addressed usability via model-driven engineering techniques to automatically transform generic feature diagrams into alloy models amenable to T-wise test generation in Alloy.

We would like to extend our work along two main dimensions. The first one concerns test generation strategies. We are currently experimenting with our toolset on a crisis management system which is characterized by a large number of optional and alternative features inducing more than one hundred billions of possible test cases for exhaustive covering. Using the incremental strategy we were able to reduce this number to a few hundred. We would also like to exploit the feature model structure to reduce the number of tuples to consider and fine-tune T-wise generation. In addition, an assessment of our generation technique with respect to greedy and meta-heuristic approaches [34], [10] would guide the tester in her toolset choices. Generated products testability is the second dimension for future work. We would like to extend our test case generation platform with automated SPL derivation techniques such as [19] acting as oracles. This will then form a complete SPL test methodology starting from considering the SPL "as a whole" to individual product testing.

\section{REFERENCES}

[1] K. Pohl, G. Böckle, and F. J. van der Linden, Software Product Line Engineering: Foundations, Principles and Techniques. Secaucus, NJ, USA: Springer-Verlag New York, Inc., 2005.

[2] P. Clements and L. Northrop, Software Product Lines: Practices and Patterns. Addison Wesley, Reading, MA, USA, 2001.

[3] K. Kang, S. Cohen, J. Hess, W. Novak, and S. Peterson, "FeatureOriented Domain Analysis (FODA) Feasibility Study," Software Engineering Institute, Tech. Rep. CMU/SEI-90-TR-21, Nov. 1990.

[4] E. Uzuncaova, D. Garcia, S. Khurshid, and D. Batory, "Testing software product lines using incremental test generation," in ISSRE. IEEE Computer Society, 2008, pp. 249-258.

[5] A. Tevanlinna, J. Taina, and R. Kauppinen, "Product family testing: a survey," SIGSOFT Softw. Eng. Notes, vol. 29, no. 2, pp. 12-12, 2004.
[6] J. McGregor, "Testing a software product line," CMU/SEI, Tech. Rep., 2001.

[7] D. M. Cohen, S. R. Dalal, M. L. Fredman, and G. C. Patton, "The AETG System: an approach to testing based on combinatorial design," IEEE Trans. Softw. Eng., vol. 23, pp. 437-444, 1997.

[8] D. R. Kuhn, D. R. Wallace, and A. M. Gallo, "Software fault interactions and implications for software testing," IEEE Trans. Softw. Eng., vol. 30, no. 6, pp. 418-421, 2004.

[9] M. B. Cohen, M. B. Dwyer, and J. Shi, "Coverage and adequacy in software product line testing," in ROSATEA@ISSTA, 2006, pp. 53-63.

[10] M. Cohen, M. Dwyer, and J. Shi, "Interaction testing of highlyconfigurable systems in the presence of constraints," in ISSTA, 2007, pp. $129-139$.

[11] "Alloy community," 2010. [Online]. Available: http://alloy.mit.edu

[12] D. Jackson, Software Abstractions: Logic, Language, and Analysis. MIT Press, March 2006.

[13] J. Kienzle, W. A. Abed, and J. Klein, "Aspect-Oriented Multi-View Modeling," in AOSD. ACM Press, March 2009, pp. 87 - 98.

[14] J. Kienzle and G. Bölükbaşi, "AspectOPTIMA: An Aspect-Oriented Framework for the Generation of Transaction Middleware," McGill University, Tech. Rep. SOCS-TR-2008.4, 2008.

[15] D. S. Batory, "Feature models, grammars, and propositional formulas," in $S P L C, 2005$, pp. 7-20.

[16] P.-Y. Schobbens, P. Heymans, J.-C. Trigaux, and Y. Bontemps, "Feature Diagrams: A Survey and A Formal Semantics," in $R E, 2006$

[17] P. Schobbens, P. Heymans, J. Trigaux, and Y. Bontemps, "Generic semantics of feature diagrams," Computer Networks, vol. 51, no. 2, pp. 456-479, 2007

[18] K. Czarnecki and A. Wasowski, "Feature diagrams and logics: There and back again," in SPLC. Los Alamitos, CA, USA: IEEE Computer Society, 2007, pp. 23-34.

[19] G. Perrouin, J. Klein, N. Guelfi, and J.-M. Jézéquel, "Reconciling automation and flexibility in product derivation," in SPLC. Limerick, Ireland: IEEE Computer Society, 2008, pp. 339-348.

[20] M. Phadke, Quality engineering using robust design. Prentice Hall PTR Upper Saddle River, NJ, USA, 1995.

[21] E. Torlak and D. Jackson, "Kodkod: A relational model finder," in Tools and Algorithms for Construction and Analysis of Systems, March 2007.

[22] Y. S. Mahajan and S. M. Z. Fu, "Zchaff2004: An efficient sat solver," in SAT 2004, 2004, pp. 360-375.

[23] Niklas Een and Niklas Sorensson, "MiniSat: A SAT Solver with Conflict-Clause Minimization, Poster," in SAT 2005, 2005.

[24] D. Benavides, A. Ruiz-Cortés, don Batory, and P. Heymans, "1st intl. workshop on analysis of software product lines ASPL'08," in SPLC. IEEE Computer Society, 2008, p. 385.

[25] M. Mendonça, A. Wasowski, and K. Czarnecki, "Sat-based analysis of feature models is easy," in SPLC, San Francisco, CA, USA, 2009.

[26] R. Monasson, R. Zecchina, S. Kirkpatrick, B. Selman, and L. Troyansky, "Determining computational complexity from characteristic phase transitions," Nature, vol. 400, no. 6740, pp. 133-137, 1999.

[27] P.-A. Muller, F. Fleurey, and J.-M. Jézéquel, "Weaving Executability into Object-Oriented Meta-Languages," in MODELS/UML. Springer, 2005.

[28] C. Nebut, Y. Le Traon, and J.-M. Jézéquel, Software Product Lines. Springer Verlag, 2006, ch. System Testing of Product Families: from Requirements to Test Cases, pp. 447-478.

[29] K. D. Scheidemann, "Optimizing the Selection of Representative Configurations in Verification of Evolving Product Lines of Distributed Embedded Systems," in SPLC, 2006, pp. 75-84.

[30] I. Yoon, A. Sussman, A. Memon, and A. Porter, "Direct-dependencybased software compatibility testing," in ASE, Atlanta, Georgia, USA, 2007, pp. 409-412.

[31] T. M. R. Gheyi and P. Borba., "A theory for feature models in alloy," in First Alloy Workshop, 2006.

[32] A. Metzger, K. Pohl, P. Heymans, P.-Y. Schobbens, and G. Saval, "Disambiguating the documentation of variability in software product lines: A separation of concerns, formalization and automated analysis," $R E$, pp. 243-253, 2007.

[33] D. Benavides, S. Segura, P. Trinidad, and A. R. Cortés, "FAMA: Tooling a Framework for the Automated Analysis of Feature Models," in VaMoS, 2007, pp. 129-134.

[34] A. Calvagna and A. Gargantini, "Combining satisfiability solving and heuristics to constrained combinatorial interaction testing," in Intl. Conference on Tests and Proofs. Berlin, Heidelberg: Springer-Verlag, 2009, pp. 27-42. 